\title{
Colopexia e deferentopexia associadas à omentopexia no tratamento da hérnia perineal em cães: um estudo de trinta casos
}

\author{
Colopexy and deferentopexy associated with omentopexy in the treatment of perineal hernia in dogs. \\ Study of thirty cases
}

\author{
Mario Jorge Melhor Heine D’Assis ${ }^{\text {** }}$ João Moreira da Costa Neto ${ }^{\text {II }}$ \\ Alessandra da Silva Estrela-LimaII Emanoel Ferreira Martins Filho ${ }^{I}$ \\ Júlia Morena de Miranda Leão Toríbio ${ }^{\mathrm{I}}$ Raquel Graça Teixeira ${ }^{\mathrm{I}}$
}

RESUMO

Diversas técnicas cirúrgicas têm sido propostas para correção da hérnia perineal em cães $e$ independentemente do método utilizado, o risco cirúrgico relacionado ao estado clínico e à idade do animal deve ser sempre considerado. O comprometimento do trato urinário e/ ou digestório é frequente, e o paciente geriátrico pode apresentar problemas múltiplos que afetam consideravelmente sua tolerância à anestesia, à cirurgia e a infecções. Nesse contexto, objetivou-se avaliar o emprego da colopexia $e$ da deferentopexia associadas à omentopexia como terapêutica cirúrgica alternativa em 30 cães idosos, comprometidos sistemicamente, portadores de hérnia perineal e anomalia retal concomitante. A análise dos casos permitiu verificar que a associação de procedimentos possibilitou a inspeção $e$ avaliação dos órgãos abdominais, particularmente os implicados como conteúdo herniário; a restauração do formato linear do colo distal, reduzindo seu diâmetro e favorecendo o retorno à função normal; fixação eficaz e segura no reposicionamento cranial da próstata e bexiga, evitando posteriores deslocamentos caudais; e satisfatória reparação tecidual e oclusão do defeito no diafragma pélvico. Dessa forma, concluiu-se que a técnica cirúrgica proposta tem significativo valor terapêutico quando empregada em portadores de hérnia perineal e saculação retal concomitante.

Palavras-chave: cirurgia, hérnia perineal, pexia, cão.

\section{ABSTRACT}

Several surgical techniques have been proposed for perineal hernia correction in dogs and regardless of the used method the surgical risk related to clinical status and animal age should always be considered, since the involvement of the urinary tract and/or digestive tract is frequent and the geriatric patient can present multiple problems that significantly affect their tolerance to anesthesia, surgery and infections. In this context, this study aimed to evaluate the use of colopexy and deferentopexy associated with omentopexy as surgical alternative in 30 elderly dogs, systemically compromised, which had perineal hernia and concomitant rectal anomaly. The cases analysis showed that the proposed surgical technique allowed the inspection and evaluation of abdominal organs, particularly those with hernial content involved; the distal colon linear format restoration, reducing its diameter and encouraging a normal function return, effective setting and secure cranial prostate and urinary bladder repositioning, avoiding further caudal displacement; satisfactory tissue repair and defect occlusion of the pelvic diaphragm. Thus, it was concluded that the proposed procedure has therapeutic value for dogs with perineal hernia and concomitant rectal sacculation.

Key words: surgery, perineal hernia, pexia, dog.

\section{INTRODUÇÃO}

Diversos procedimentos cirúrgicos individualizados ou simultâneos têm sido propostos para correção da hérnia perineal em cães. A maioria deles preconiza a abordagem cirúrgica via região perineal para redução do conteúdo herniário e reparação do diafragma pélvico, empregando métodos como síntese associada ou não a transposições musculares (VAN SLUIJS \& SJOLLEMA, 1989); enxertos sintéticos (MATERA et al., 1981) ou biológicos (DALECK et al., 1992). Entretanto, tais métodos frequentemente estão relacionados à dificuldade de execução, a altas taxas de recorrência e a complicações pós-operatórias (VAN SLUIJS \& SJOLLEMA, 1989; BELLENGER \& CANFIELD, 1998; FERREIRA \& DELGADO, 2003).

IPrograma de Pós-graduação em Ciência Animal nos Trópicos, Escola de Medicina Veterinária, Universidade Federal da Bahia (UFBA), 40170-110, Salvador, BA, Brasil. E-mail: mariojorgeh@uol.com.br. *Autor para correspondência.

IIDepartamento de Patologia e Clínicas, Faculdade de Medicina Veterinária, UFBA, Salvador, BA, Brasil. 
O comprometimento de órgãos do sistema digestório e/ou genito-urinário provoca uma série de alterações sistêmicas que elevam o risco operatório. A coexistência de anomalias retais, como saculação e divertículo, são fatores que podem comprometer a eficácia da herniorrafia e também devem sofrer correção cirúrgica (COSTANETO et al., 2006; SOUZA \& ABÍLIO, 2007). Deve-se considerar a predisposição em animais geriatras, que possuem baixa tolerância a anestesia, cirurgia e infecções (SOUZA \& ABILIO, 2007).

A partir dos promissores resultados obtidos com o emprego da deferentopexia ou cistopexia e colopexia no tratamento da herniorrafia perineal, abordagens abdominais têm sido propostas com o intuito de avaliar e reparar os órgãos envolvidos, bem como corrigir transtornos causados pela projeção desses órgãos no diafragma pélvico (BILBREY et al., 1990; BRISSOT et al., 2004).

O reposicionamento vesical e prostático conseguido mediante a deferentopexia (BILBREY et al., 1990; BRISSOT et al., 2004; SEIM III, 2004; BARREAU, 2008) é indicado para minimizar a pressão sobre o diafragma pélvico e prevenir posteriores deslocamentos caudais dessas vísceras, diminuindo o risco de recidiva. Paralelamente, a colopexia também se mostrou efetiva na redução de anomalias, como saculações, desvios ou divertículos retais, reduzindo o diâmetro retal e restaurando o formato linear do colo (BRISSOT et al., 2004; SEIM III, 2004).

Estudos relatam a utilização, com sucesso, da omentopexia em determinados procedimentos cirúrgicos (RUFFINI, 1992). As propriedades terapêuticas do omento se devem a sua capacidade de induzir reparação a partir da revascularização tecidual e atuar como barreira física, protegendo as estruturas (BRISSOT et al. 2004). Esses fatores podem contribuir significativamente para uma melhor e mais rápida resolução de determinadas patologias.

A deferentopexia e a colopexia podem ser empregadas como tratamento único ou complementar à herniorrafia perineal, principalmente em casos bilaterais, de grandes dimensões ou recidiva, com grandes benefícios terapêuticos e poucas complicações (BARREAU, 2008).

Neste estudo, objetivou-se avaliar o emprego da colopexia e deferentopexia associadas à omentopexia como terapêutica cirúrgica alternativa em portadores de hérnia perineal e anomalia retal concomitante.

\section{MATERIAL E MÉTODOS}

No período compreendido entre maio de 2004 e outubro de 2008, foram atendidos, no Hospital de Medicina Veterinária da Universidade Federal da Bahia, 67 cães, todos machos, portadores de hérnia perineal, dentre os quais 30 foram selecionados para o desenvolvimento desta pesquisa. Os critérios para seleção dos animais se basearam na idade dos pacientes (superior a sete anos) e na presença de alterações sistêmicas que os classificavam, segundo PITREZ \& PIONER (2003), em pacientes de elevado risco cirúrgico. Foram avaliadas características clínicas relacionadas ao aumento de volume da região perineal, à presença e ao tipo de conteúdo herniário, redutível ou não, e à presença de anomalias retais. As alterações sistêmicas resultantes direta ou indiretamente da hérnia perineal foram confirmadas a partir de exames laboratoriais complementares relacionados à função renal e hepática (hemograma completo e bioquímicos).

A terapêutica clínico-cirúrgica incluiu estabilização prévia do paciente. Animais com retroflexão de bexiga foram sondados para esvaziamento vesical. Na impossibilidade de sondagem imediata, efetuava-se inicialmente, sob rigorosa técnica asséptica, cistocentese por via perineal, drenagem parcial de urina e posteriormente sondagem, até o total esvaziamento vesical. Realizava-se recondução do conteúdo herniário à cavidade abdominal e fluidoterapia de suporte com solução fisiológica 0,9\% ou ringer com lactato por período mínimo de 12 horas. Foi instituída terapia antimicrobiana profilática à base de enrofloxacina ( $5 \mathrm{mg} \mathrm{kg}^{-1}$ a cada $12 \mathrm{~h}$ ) ou cefalexina (25 mg kg ${ }^{-1}$ a cada $12 \mathrm{~h}$ ), em associação com metronidazol (15 $\mathrm{mg} \mathrm{kg}^{-1}$ a cada $\left.12 \mathrm{~h}\right)$ e terapia anti-inflamatória à base de meloxicam ( $0,1 \mathrm{mg} \mathrm{kg}^{-1}$ a cada $\left.24 \mathrm{~h}\right)$.

Os casos de retenção fecal, particularmente relacionados com saculação ou desvio retal, foram tratados mediante lavagem intestinal utilizando solução salina morna associada à glicerina. Após fluidoterapia, recomendava-se dieta líquida/pastosa rica em fibras e proteínas e administração de soro caseiro ad libitum. Os animais foram acompanhados diariamente, e os procedimentos cirúrgicos efetuados no prazo máximo de 72 horas após o atendimento.

Sessenta minutos antes do procedimento cirúrgico, os animais receberam medicação antimicrobiana (enrofloxacina $5 \mathrm{mg} \mathrm{kg}^{-1}$ ) por via intramuscular. O protocolo anestésico incluiu a associação de cloridrato de acepromazina $\left(0,05 \mathrm{mg} \mathrm{kg}^{-1}\right)$ e cloridrato de tramadol ( $\left.2 \mathrm{mg} \mathrm{kg}^{-1}\right)$, por via endovenosa como medicação pré-anestésica, seguido de indução anestésica com propofol (5-10 $\mathrm{mg} \mathrm{kg}^{-1}$ ) e manutenção anestésica com isoflurano em circuito semiaberto.

O procedimento cirúrgico teve início pela orquiectomia aberta mediante incisão mediana préescrotal ou escrotal (a depender do peso do animal), 
exteriorização e exposição dos testículos. Posteriormente, realizou-se incisão da túnica vaginal parietal, estendendo-se no sentido do cordão espermático com identificação dos componentes testiculares. O ducto deferente foi localizado e cuidadosamente separado do plexo pampiniforme. Sua obliteração foi feita individualmente, por meio de ligadura na sua porção distal, sendo empregados fios cirúrgicos de náilon 2-0 e deixando-se os segmentos dos fios longos. O plexo pampiniforme foi obliterado por meio de ligadura na sua porção cranial, empregando-se fios categute cromado 2-0. Em seguida, foi realizada a ablação do testículo, permanecendo-se apenas com o ducto deferente. O mesmo procedimento foi realizado no testículo contralateral. A orquiectomia pré-escrotal foi fechada com dupla sutura, utilizando catgute 2-0 subcutâneo e náilon 2-0 na pele, enquanto que a orquiectomia escrotal foi deixada aberta para cicatrização por segunda intenção.

Após a orquiectomia, iniciou-se procedimento cirúrgico para celiotomia, empregandose incisão mediana ventral retro-umbilical. Após inspeção da cavidade abdominal caudal, foram identificados o colo, a bexiga, a próstata e os respectivos ductos deferentes. Os ductos foram moderadamente tracionados por meio dos canais inguinais, para que penetrassem na cavidade abdominal, sendo, nesse momento, dissecados das adesões peritoniais até o nível da glândula prostática em sua posição anatômica. Ao término dessa fase, iniciou-se a colopexia.

Para realização da colopexia, a porção final do colo foi tracionada cranialmente, sendo estimada uma área de adesão entre sua borda antimesentérica e a parede abdominal média latero-dorsal esquerda. Nessa área, após escarificação da serosa do colo e incisão transversal no músculo transverso abdominal, realizouse a pexia, empregando-se sutura de Wolf, em dupla camada, com fios de náilon 2-0.

Em seguida, deu-se inicio à deferentopexia, baseada na técnica proposta por BILBREY et al. (1990). Moderada tração cranial foi aplicada em cada ducto deferente para tracionar a bexiga e próstata. Foram feitas duas incisões paralelas no músculo transverso e, com auxílio de uma pinça hemostática de crile curva, criouse um túnel sob esse músculo, em direção craniocaudal. O ducto deferente foi pinçado, tracionado sob o túnel e rebatido por sobre ele, sendo fixado por meio de sutura em " $X$ ” à parede abdominal, sendo utilizados fios de náilon 2-0 ou 0 . Procedimento idêntico foi realizado no lado contralateral.

A omentopexia foi efetuada com retalho pediculado de omento maior, que recobriu a bexiga e próstata e foi fixado ao diafragma pélvico, por meio de pontos simples separados, utilizando-se fio de náilon 2-0, de forma a obliterar a falha muscular.

A síntese da cavidade abdominal foi realizada com fio absorvível, sendo empregadas sutura em “ $X$ ” para parede abdominal, sutura intradérmica e sutura isolada simples com fio de náilon para síntese de pele.

No pós-operatório, foi mantida a antibioticoterapia anteriormente prescrita por um período de sete a 10 dias, como também a terapia antiinflamatória. Além disso, também instituiu-se terapia analgésica com cloridrato de tramadol por via oral na dose de $2 \mathrm{mg} \mathrm{kg}^{-1}$, a cada 12 horas, por três dias. Além disso, foram indicados dieta rica em fibras, uso de lubrificantes intestinais ou emolientes fecais, método de restrição e curativo local até a retirada dos pontos cutâneas, no $10^{\circ}$ dia pós-operatório.

Avaliações clínicas foram feitas aos três, 10 , 30 e 60 dias de pós-cirúrgico. Após esse período, informações foram adquiridas por via telefônica ou visitas esporádicas. Os proprietários e/ou responsáveis foram questionados quanto ao comportamento do animal, às reações dolorosas, às atitudes de postura ao defecar e urinar e ao aspecto, à coloração e ao volume das fezes e da urina. Os animais foram acompanhados por um período de até três anos.

\section{RESULTADOS E DISCUSSÃO}

Todos os animais estudados apresentavam alterações sistêmicas referentes ao quadro de obstrução urinária com consequente injúria renal, condição que não recomendava a realização de procedimentos cirúrgicos longos e complexos relacionados à reparação do diafragma pélvico e serviram de objeto de estudo para avaliação da técnica proposta.

O tempo de acompanhamento variou de 30 dias a três anos, com média de 11 meses. A faixa etária incluiu animais de sete a 14 anos, sendo a média de idade dos animais estudados igual a 10 anos. Esses achados corroboram a literatura consultada, que classifica a hérnia perineal como doença frequente em animais idosos (MORTARI \& RAHAL, 2005; BARREAU, 2008). O paciente geriátrico, com certa frequência, apresenta problemas múltiplos que podem afetar significativamente a sua tolerância à anestesia e cirurgia (HOSGOOD et al., 1995; SOUZA \& ABÍLIO, 2007). Em concordância, os resultados obtidos neste estudo, a partir da avaliação clínica-cirurgica dos pacientes, indicaram que o envelhecimento é um processo que reduz a capacidade do indivíduo de manter a homeostasia durante estresses fisiológicos 
internos e ambientais externos, acarretando maior vulnerabilidade a doenças com uma menor resposta orgânica. Essas peculiaridades apontam com clareza a extrema cautela que deve cercar a avaliação desses pacientes, quando candidatos a cirurgias mais complexas.

O aumento de volume perineal foi observado bilateralmente em 19 casos (63,27\%), unilateral direito em seis casos (19,98\%) e em cinco casos unilateral esquerdo (16,65\%). Diante do significativo percentual de hérnias unilaterais (36,63\%), verificou-se que o procedimento cirúrgico proposto também minimizou o risco de nova formação herniária no lado contralateral. Fato frequentemente observado pós redução unilateral em razão da fragilidade muscular na região contralateral, resultando em maior predisposição à ocorrência dessa alteração (RAISER, 1994; COSTA NETO et al., 2006).

A retroflexão da bexiga é uma condição que causa abrupta curvatura uretral, provocando oclusão parcial ou total do fluxo urinário, distensão vesical, comprometimento do suprimento neurovascular e atonia, com consequente elevação das concentrações séricas de ureia e creatinina e dos índices de morbidade e mortalidade dos cães com hérnia perineal (WHITE \& HERRTAGE, 1986; HOSGOOD et al., 1995). Tal alteração foi observada em 26 animais (86,58\%), resultando em significativa complicação sistêmica a partir de modificações no padrão bioquímico renal que sabidamente elevam o risco cirúrgico (PITREZ \& PIONER, 2003).

Neste estudo, não foi possível esclarecer a divergência literária acerca da etiologia da anomalia retal, se primária ou secundária ao processo herniário. O tenesmo, que é relacionado à prostatomegalia, conforme BELLENGER \& CANFIELD (1998), e às dilatações retais, segundo RAISER (1994), parece ser fator contribuinte em ambas as afecções, mas nem todo o portador de hérnia perineal tem hiperplasia prostática (MAUTE et al., 2001) e saculação retal, sendo esta a alteração mais relacionada a hérnias bem desenvolvidas, segundo DALECK et al. (1992). Por outro lado, KRAHWINKEL (1983) destaca que a fragilidade da musculatura perineal lesionada propicia a formação da saculação retal, porém por muitas vezes a saculação retal é encontrada sem que o animal apresente hérnia perineal.

O protocolo de emergência preconizado no primeiro atendimento, para desobstrução urinária, mostrou-se eficiente para estabilização do paciente. A drenagem da urina, como medida prioritária, conforme relata WHITE \& HERRTAGE (1986), foi obtida mediante sondagem vesical, ao contrário do que é preconizado por PARKS (1981), em que a cistocentese figura como única opção viável. Tal manobra foi realizada somente em seis animais (19,98\%), anteriormente à sondagem uretral para completo esvaziamento vesical e reposicionamento da bexiga.

A orquiectomia foi realizada em todos os animais, com objetivo de reduzir os riscos de reincidência em razão de um dos fatores etiológicos propostos por DALECK et al. (1992), que reconhecem o envolvimento da próstata hipertrofiada nos animais com hérnia perineal. Realizada em uma primeira fase do procedimento cirúrgico, a orquiectomia permitiu a preparação dos ductos deferentes para posterior fixação intra-abdominal. A permanência dos segmentos de fios longos deixados no momento da obliteração distal auxiliou a visualização e a execução de manobras referentes à deferentopexia.

$\mathrm{O}$ acesso cirúrgico via abdominal mediano ventral, assim como também evidenciado por BILBREY et al. (1990), BRISSOT et al. (2004) e BARREAU (2008), permitiu a inspeção e avaliação mais acurada da cavidade abdominal e de seus órgãos, particularmente a bexiga e a próstata, evidenciando lesões deletérias causadas pela retroflexão, compressão e permanência desses órgãos como conteúdo herniário. Foram observados abscessos prostáticos em dois casos, e um deles apresentava peritonite severa tratado com copiosa lavagem abdominal utilizando solução fisiológica. Em dois casos, neoplasias foram identificadas comprometendo baço e rim, sendo realizada a exérese destes.

A tração cranial do colo, obtida pela colopexia (Figura 1B), em um primeiro momento, proporcionou a restauração do seu formato linear, reduzindo seu lúmen e proporcionando satisfatória evacuação. Porém, no pós-operatório tardio, notouse, em 13 casos (43,29\%), nova dilatação em colo distal, além da persistência do tenesmo, também evidenciado por SEIM III (2004), que observou essa persistência em 55\% dos casos. Tais achados caracterizam a técnica como paliativa; porém, em virtude da característica clínica dos pacientes, pode ser empregada por minimizar os sintomas e permitir o emprego de terapias alternativas, como manejo dietético e uso de lubrificantes intestinais.

A deferentopexia (Figura 1C) mostrou-se eficaz e segura no reposicionamento e na fixação da próstata e bexiga sem apresentar riscos de complicações referentes a infecção local, à laceração de parede vesical, à formação de cálculos e à interferência na contração vesical, notadas na cistopexia por BILBREY et al. (1990). Após o reposicionamento vesical, em concordância com BARREAU (2008), todos os animais demonstraram 


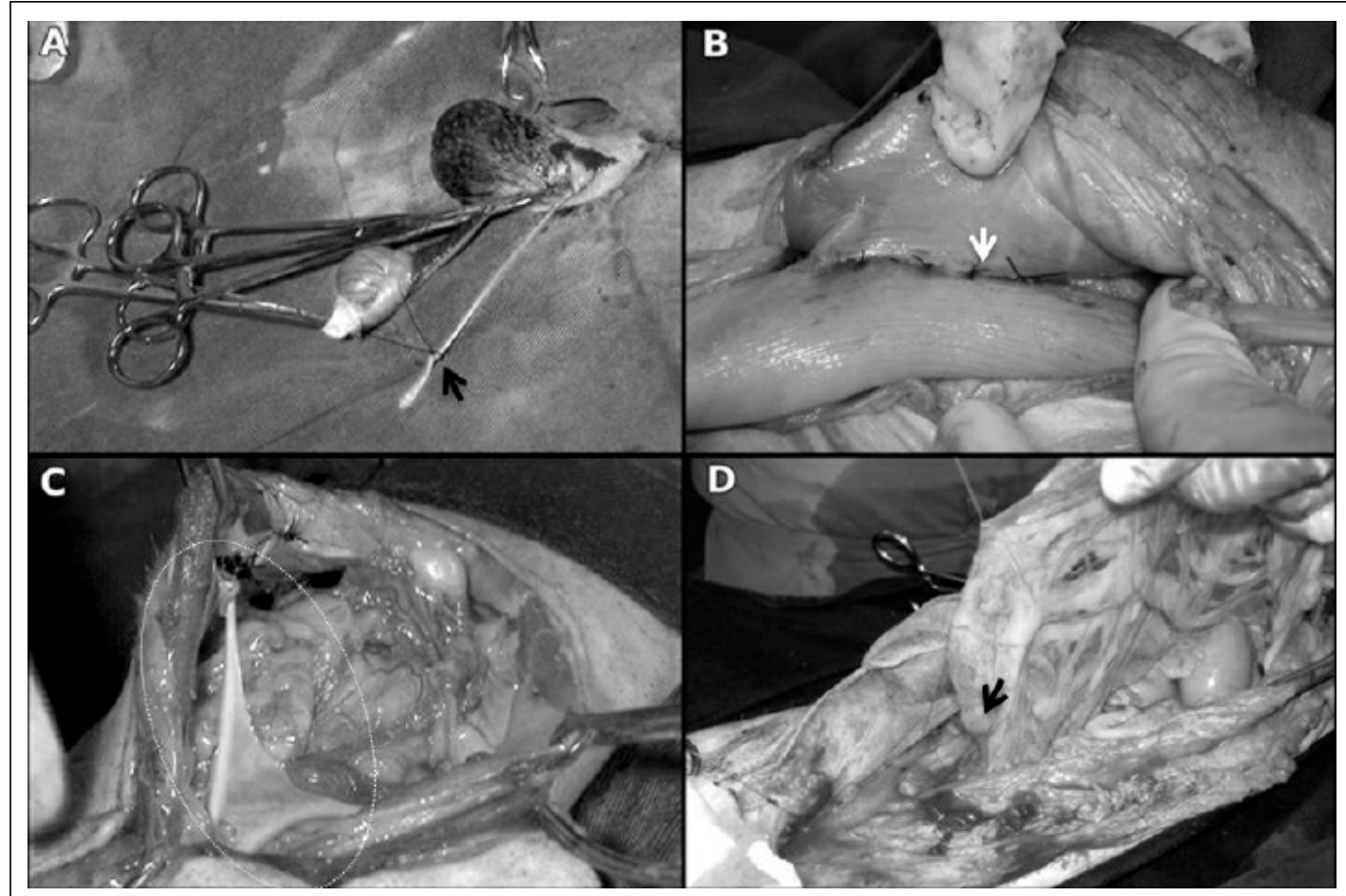

Figura 1 - Colopexia e deferentopexia associadas à omentopexia no tratamento da hérnia perineal em cães. Em A, ducto deferente preservado durante orquiectomia (seta). Em B, aspecto final da colopexia após realização de sutura de Wolf em dupla camada (seta). Em C, aspecto final da deferentopexia após fixação abdominal bilateral (área tracejada). Em D, preparação de segmento de omento maior para oclusão do diafragma pélvico e omentalização dos órgãos.

micção satisfatória, sendo a incontinência urinária observada em oito casos (26,64\%). No vigésimo dia de pós-cirúrgico, em apenas um caso (que já apresentava o sinal clínico no momento do primeiro atendimento), o quadro persistiu. Cães com retroflexão de bexiga podem desenvolver atonia ou incontinência urinária, transitória ou permanente, dependendo da severidade do dano causado aos nervos e vasos pélvicos, ao tempo de compressão e à distensão da bexiga (MORTARI \& RAHAL, 2005).

A utilização do omento para recobrimento das vísceras e preenchimento do canal pélvico (Figura 1D) baseou-se nos referenciais consultados e corroborou os resultados desses estudos (RUFFINI, 1992; BRISSOT et al., 2004), os quais sugerem que suas propriedades podem ser úteis em procedimentos cirúrgicos nos quais sejam necessários o favorecimento de aderências, a angiogênese, a drenagem linfática, o combate à infecção e a reconstituição de tecidos. Tais características terapêuticas mostraram-se fundamentais na técnica cirúrgica proposta, não só para a oclusão do defeito no diafragma pélvico, mas também para promoção da neovascularização e reparação dos órgãos envolvidos, particularmente da bexiga, assim como para propiciar a drenagem linfática da região pélvica e auxiliar a regressão do processo inflamatório local.

O reposicionamento cranial da bexiga e da próstata, conseguido mediante a fixação dos ductos deferentes à parede abdominal lateral, como observado por BILBREY et al. (1990), BRISSOT et al. (2004) e BARREAU (2008), preveniu posteriores deslocamentos caudais dessas vísceras, minimizando a pressão exercida no diafragma pélvico e diminuindo o risco de recidiva. Um dado importante não citado na literatura consultada é a persistência do volume na região perineal em razão da flacidez da pele nas hérnias bem desenvolvidas. Esse defeito estético diminuiu gradativamente ao longo do período pós-operatório, evoluindo para um resultado satisfatório, sem nenhum comprometimento sistêmico.

O procedimento cirúrgico proposto mostrou-se de fácil operacionalidade e rápida execução, não necessitando de instrumentos especiais ou de manobras complexas para sua realização. As complicações observadas no pós-operatório (deiscência de sutura, incontinência urinária e tenesmo) foram discretas e menos significativas do que aquelas 
encontradas por outros métodos de reparação perineal. A infecção e deiscência da sutura, complicações comuns nas herniorrafias perineais segundo BELLENGER (1980), incriminadas pela proximidade da ferida cirúrgica com o ânus, não foram evidenciadas. $\mathrm{O}$ único caso de deiscência observado resultou da automutilação, e a infecção não esteve presente. Prolapso retal, incontinência fecal e disúria não foram observados. A persistência do tenesmo, complicação pós-operatória frequente em herniorrafias perineais, foi tratada por meio de manejo clínico.

As taxas de recorrência da hérnia perineal, após herniorrafia perineal, variam, segundo SOUZA \& ABÍLIO (2007), entre 0 e 46\%. Nesta pesquisa, houve quatro casos a recorrência $(13,32 \%)$, e dois animais apresentaram nova retroflexão de bexiga no $15^{\circ}$ e no $28^{\circ}$ dia após a cirurgia, respectivamente, e em um dos casos identificou-se, em nova celiotomia, a ruptura dos ductos deferentes. Outros dois animais, portadores de hérnia unilateral, 30 e 120 dias após a cirurgia, respectivamente, apresentaram nova herniação no lado contra lateral. Para reversão desse quadro, empregouse a herniorrafia perineal com transposição do músculo obturador interno, conforme preconiza VAN SLUIJS \& SJOLLEMA(1989). Tal evidência fortalece a afirmativa de que a técnica proposta também pode ser empregada como tratamento preliminar à correção do diafragma pélvico, conforme cita BRISSOT et al. (2004).

\section{CONCLUSÃO}

De acordo com os resultados obtidos neste trabalho, pode-se concluir que a colopexia e deferentopexia associadas à omentalização mostramse de fácil operacionalidade e rápida execução, apresentando significativo valor terapêutico no tratamento de cães portadores de hérnia perineal, com predisposição à retroflexão de bexiga e/ou próstata e saculação retal concomitante.

\section{REFERÊNCIAS}

BARREAU, P. Perineal hernia: three steps in one surgery: pexy, sterilisation, repair. In: WORLD CONGRESS IN SMALL ANIMAL VETERINARY MEDICINE, 33., 2008, Dublin. Proceedings... Dublin: WSAVA, 2008. Online. Disponível e m : < h t t p : / / w w w . vi n . c o m / p r o c e ed in g s / Proceedings.plx?CID=WSAVA2008\&PID=23885\&O=Generic $>$. Acesso em 12 out. 2008.

BELlENGER, C.R. Perineal hérnia in dogs. Australian Veterinary Journal, v.56, p.434-438, 1980.
BELLENGER, C.R.; CANFIELD, R.B. Hérnia perineal. In: SLATTER, D. Manual de cirurgia de pequenos animais. São Paulo: Manole, 1998. p.578-590.

BILBREY, S.A. et al. Fixation of the deferent ducts for retrodisplacement of the urinary bladder and prostate in canine perineal hernia. Veterinary Surgery, v.19, n.1, p.24-27, 1990.

BRISSOT, H.N. et al. Use of laparotomy in a staged approach for resolution of bilateral or complicated perineal hernia in 41 dogs. Veterinary Surgery, v.33, p.412-421, 2004. Disponível em: <http://www3.interscience.wiley.com/journal/118771339/ abstract?CRETRY=1\&SRETRY=0>. Acesso em: 13 ago. 2008. doi: 10.1111/j.1532-950X.2004.04060.x

COSTA NETO, J.M. et al. Tratamento cirúrgico para correção de hérnia perineal em cão com saculação retal coexistente. Revista Brasileira de Produção e Saúde Animal, v.7, n.1, p. 07-19, 2006.

DALECK, C.R. et al. Reparação de hérnia perineal em cães com peritônio de bovino conservado em glicerina. Ciência Rural, v.2, n.22, p.179-183, 1992.

FERREIRA, F; DELGADO, E. Hérnias perineais nos pequenos animais. Revista Portuguesa de Ciências Veterinárias, v.545, p.3-9, 2003.

HOSGOOD, G. et al. Perineal herniorrhaphy: perioperative data from 100 dogs. Journal of the American Animal Hospital Association, v.31, n.4, p.331-341, 1995.

KRAHWINKEL, D.J. Rectal diseases and their role in perineal hernia. Veterinary Surgery, v.12, n.3, p.160-165, 1983.

MATERA, A. et al. Hérnia perineal no cão. Tratamento cirúrgico mediante utilização de malha de polipropileno. Revista da Faculdade de Medicina Veterinária e Zootecnia da Universidade de são Paulo, v.18, n.1, p.3741, 1981.

MAUTE, A.M. et al. Perineal hernie beim hund-Colopexie, vasopexie, cystopexie und kastration als therapie der wahl bei 32 hunden. Shweiz Arch Tierheilkd, v.149, p.360-367, 2001.

MORTARI, A.C.; RAHAL, S.C. Hérnia perineal em cães. Ciência Rural, v.35, n.5, p.1220-1228, 2005. Disponível em: $<$ http://www.scielo.br/scielo.php?pid=S 0103 $84782005000500040 \&$ script $=$ sci_arttext $\&$ tlng $=$ en $>$. Acesso em: 08 jun. 2008. doi: 10.1590/S0103-84782005000500040

PARKS, J. Herniation. In: BOJRAB, M.J. Pathophysiology in small animal surgery. Philadelphia: Lea \& Febeger, 1981. p.420-423.

PITREZ, F.A.B.; PIONER, S.R. Avaliação e preparo préoperatórios. In: PITREZ, F.A.B.; PIONER, S.R. Pré e pósoperatório em cirurgia geral e especializada. 2.ed. Porto Alegre: Artmed, 2003. p.31-41. 
RAISER, A.G. Herniorrafia perineal em cães - análise de 35casos. Brazilian Journal of Veterinary Research and Animal Science. v.31, n.3/4, p.252-260, 1994.

RUFFINI, E. Surgical aplications of the greater omentum: a critical review of the literature. PanMinerva Medicine, v.34, p.135-140, 1992

SEIM III, H.B. Perineal hernia repair. In: WORLD CONGRESS IN SMALL ANIMAL VETERINARY MEDICINE, 29., 2004, Rhodes. Procedings... Rhodes: Alta Grafico Publisher, 2004. V.1, p.833-836.
SOUZA, D.B.; ABÍLIO, J.A. Hérnia perineal em cães- revisão de literatura, Revista Clínica Veterinária, n.68, p.78-86, 2007.

VAN SLUIJS, F.J.; SJOLLEMA, B.E. Perineal hérnia repair in the dog by transposition of the internal obturator muscle. Surgery technique. Veterinary Quartely, v.11, n.1, p.13-17, 1989.

WHITE, R.A.S.; HERRTAGE, M.E. Bladder retroflexion in the dog. Journal Small Animal Practice, v.27, p. 735-746, 1986. Disponível em: <http://www3.interscience.wiley.com/ journal/119490795/abstract?CRETRY $=1 \& S R E T R Y=>$. doi: 10.1111/j.1748-5827.1986.tb02116.x. 